\title{
Questions Posed by the Use of Genetic Information for Personalized Medicine Achievements and Promises
}

\author{
George N Goulielmos ${ }^{1 *}$, Maria I Zervou ${ }^{1}$, Agata Burska ${ }^{2}$ and Frederique Ponchel ${ }^{2}$ \\ ${ }^{1}$ Laboratory of Molecular Medicine and Human Genetics, Department of Internal Medicine, Medical School of Crete, Heraklion, Greece
}

${ }^{2}$ Leeds Institute of Rheumatic and Musculoskeletal Medicine, The University of Leeds, Leeds, UK

*Corresponding author: George N Goulielmos, Department of Medicine, Voutes, 71500 Heraklion, Crete, Greece, and Tel: +302810394626, E-mail: goulielmos@med.uoc.gr

Received date: December 01, 2015; Accepted date: January 19, 2016; Published date: January 21, 2016

Copyright: (c) 2016 Goulielmos GN, et al. This is an open-access article distributed under the terms of the Creative Commons Attribution License, which permits unrestricted use, distribution, and reproduction in any medium, provided the original author and source are credited.

\begin{abstract}
Personalised medicine (PM) has the potential to increase therapeutic effectiveness, reduce side effects and lower cost. The identification of biomarkers predictive of the clinical response to specific treatments in subsets of patients became reality for a variety of diseases. However, a better understanding of the benefits and limitations needs to be developed at the level of the general public as well as at the level of an individual patient. The upcoming ability to characterize each patient from the genetic point of view in a comprehensive manner is believed to have the potential to transform medicine, thus enabling accurate prognosis as well as a treatment outcome prediction. However, PM holds both promise and cause for concern. Although PM promises that an individual's genetic information may be increasingly used to prioritize medical decision making, it raises in parallel fears and questions as to whether such use could be inequitable. Thus, there are many thoughts whether the use of individual genetic information in the delivery of health care can be a cause for concern, as it may lead to genetic discrimination and other problems such as with employers and private insurance companies. Finally, the main pitfall of predictive tests for complex disease remains the putative lack of proven medical benefit. A better understanding of the benefits PM will have to be developed at the level of the general public as well as at the level of an individual patient; which will also reassure people that their genetic data is used appropriately to choose therapeutic protocols and drugs.
\end{abstract}

Keywords: Personalized medicine; Pharmacogenetics; Genetic discrimination; Biomarkers; Ethics

\section{Introduction}

Precision or Personalized medicine (PM) proposes to customise healthcare, with respect to medical decisions, practices, and/or products by tailoring these to the individual patient. In this context, genetic testing is often employed for selecting appropriate and optimal therapies based on a patient's genetic make-up. PM has the potential to revolutionize medical care by utilizing an improved understanding of genetics to allow for better diagnostic tests, greater predictability of disease course and improved patient safety by selecting not only the right drug for the right patient but also the proper dosage and timing to reduce adverse effects. Such achievements have been made possible by the recent advances of biotechnological sciences generating diseaserelevant molecular information, with the potential for tailoring patient care. We will limit our discussion to genetics data however, several other fields (notably all-omics) have massively progressed over the past few years. And nowadays challenges may lie more in establishing frameworks for regulating, compiling, and interpreting the influx of information.

The use of genetic data in PM has first taken off in oncology where treating malignancies with specific drugs was based on genetic biomarkers or other tumor characteristics. Thus, the identification of biomarkers predictive of the clinical response to specific treatments in subsets of patients allowed PM to be initiated for a few diseases. The development of PM, apart from the challenges and promises it brings, raises concerns that should be addressed before its overall vision comes true. According to the principle of PM, a particular treatment will be given only to patients that are the most likely to benefit from it. The possibility to tailor therapies specifically for each patient revealed the necessity to fill the gap in the awareness and understanding by patients of the concept involved in PM for them to comprehend why they are (or not) getting certain treatment.

Genetics in the public eye had a bad reputation mostly linked to historical facts and eugenics concepts. The development of a communication campaign by the Human Genome project (http:// en.wikipedia.org/wiki/Human_Genome_Project; www.genome.gov/ 12011239; http://plato.stanford.edu/entries/human-genome/) has greatly contributed to reducing fears and moved the discussion to an ethical and legal level. Genetic discrimination remains today's most obvious general public concern while access to genetics information hence to PM represents the other side of the conundrum [1]. The collection, records and use of genetic information had nonetheless raised major concerns, if not misconceptions related to discrimination and stigmatization. Legal and ethical concerns mostly revolving around issue related to genetic discrimination have predominantly focused around employment or private insurance, suggesting that there is a need to consider a broader view of genetic discrimination, departing from simple categorization [2].

\section{Discrimination}

Historically, human variation has been classified by assigning subjects to different races. Any tailoring of medical treatment based on race is however recipe for failure considering that ethnicity has a poor relationship to health. Whether states, employers or insurers should be 
allowed to take into account genetically derived information that reveals an individual's prospects for future disease and incapacity is the main point of concern. This is perceived to have the potential for impacting on all sorts of societal and socio-economical factors from employment to the purchase of health, life or disability insurance. It is conceivable that employers may claim to have a legitimate financial interest in genetic data about future potential employees, given that those at increased risk for a genetic disorder may generate expenses such as sick days. However, this may further affect decisions with regards to promotion, provision of additional training, disability pay and pensions, which are non-ethical even illegal, as there are clear laws forbidding genetic discrimination in the workplace (ban genetic discrimination in the workplace) [3].

\section{Health insurance}

The other main concern is about using genetic data in relation to insurance with the fear that genetic information can be used by insurers to deny, limit or cancel health insurance policies (for example in the US http://ghr.nlm.nih.gov/handbook/testing/ insurancecoverage). Despite the confidence of the public that genetic research has brought health benefit, this is nonetheless causing some patients to refuse to participate in research studies while others refuse to have any genetic test even when advised to due to a possible genetic disorder.

Concerns were also voiced when insurers express the willingness to fund the genomic revolution and any validation required to define criteria for reimbursement. At this stage, it was still unclear how companies are navigating their way through the world's regulatory bodies. It is likely that people would avoid genetic testing because of fear of losing their policy $[3,4]$. Insurers have asserted that they would not perform tests to obtain specific genetic information but argued that they should be free to use available information, citing the need to avoid "moral hazard" (the risk that people who know they will become ill will try to obtain insurance at regular rates) [5].

Taking the example of breast and ovarian cancer, risk counselling based on a genetic test for the Breast Cancer Risk gene (BRAC-1), raised concerns about confidentiality and the potential for discrimination prevented $20-25 \%$ of eligible women from undertaking the test [6]. Analysing the reason for refusing the test, over $50 \%$ of women asked cited concern about insurance discrimination. Although, example of discrimination based on genetic test results could not be documented, there have been other negative insurance-related experiences [7], and legislation was established in several (but not all) countries [8] (i.e., Germany Genetic Diagnosis Act in 2009) towards limiting the use of genetic information by insurances companies.

\section{Legal constraints}

The debates initiated by the insurance related issues are still continuing and more laws, bills and agreements have and keep being produced in response to public pressure, notably in the US [9] with bills being introduced in many sessions of the Congress (Genetic Information Non-discrimination in Health Act of 2003, S. 1053, $108^{\text {th }}$ Cong.) and optimal solutions are still being thought. In Europe, the regulatory control of genetic testing was enacted in 2008 (Additional Protocol to the 1997 Convention for the Protection of Human Rights and Dignity of the Human Being) with regard to the application of Biology and Genetic Testing in Medicine, for Health Purposes. Although not currently binding, it represents the first European legal instrument in this area. The original European Convention on Human Rights and Biomedicine states, in Article 12, that 'tests which are predictive of genetic diseases or which serve either to identify the subject as a carrier of a gene responsible for a disease or to detect a genetic predisposition or susceptibility to a disease may be performed only for health purposes or for scientific research linked to health purposes, and subject to appropriate genetic counseling. The Additional Protocol on genetic testing in 2008 further stated in article $7 \S 1$ that 'a genetic test for health purposes may only be performed under individualized medical supervision' [10].

In the context of PM, it is likely that regulatory element will be needed in the future to optimise the use of such tools. Currently, the regulation of clinical trials to establish the value of PM is notably slowed down by an excess of lengthy approval processes. In our economical context, is it critical to allow PM to develop rapidly, streamlining approval processes notably as patients stratification should take all into account previous experience acquired for a particular drug in a particular disease. The focus on clinical effectiveness associated with the stratification should be paralleled by economical gain.

\section{Relatives of patients}

Another important concern refers to patient's relatives, a problem that cannot be solved easily due to the lack of common views on how to handle the ethical difficulties involved. In any case, professional involvement is indicated for any type of genetic discussion involving minors [11]. Genetic testing for clinical purposed should only take place through a healthcare provider and with adequate counselling. It is particularly discouraged to use home-kit genetic testing (available for certain diseases) to assess a "risk to develop" a disease (see section below) and notably on children. In addition to the risks of inaccurate results, inaccurate interpretations, potentially harmful interventions and alteration in family dynamics will most likely results from this with negative consequences $[12,13]$. Furthermore, in the context of PM tests, it is important to get across the message that a test is only valid for a certain time in the course of a disease and only reflect a patient's history up to that point in time (notably taking in consideration previously failed treatment options). Such test would therefore not be suitable for a family member notably if this person has not yet developed the disease itself even if tested positive in a risk-assessment. At this stage, lifestyle modification and preventive measures (if available) are more likely to be effective.

\section{Incidental findings}

Incidental findings are information that could have important impact on patients but that were not the primary reason for performing the test. Any test that produces genetic information will tend to produce incidental findings because more may be visible than what was originally sought. Systematic reviews of the ethical reasons presented in the literature for and against the disclosure of incidental findings arising in genetic research contexts were conducted [14]. The view defended by those favoring disclosure, is that only incidental findings, that have a confirmed clinical utility (i.e., possibility of treatment or prevention) should be disclosed to patient by qualified professionals. A second recommendation related to minors suggested proceeding with caution especially when using new technologies. It is also recommended that the number of possible incidental findings be limited even before genetic testing is carried out. 


\section{Commercial use}

PM has the potential to select the right drug for the right patient at the right time during his/her disease course. A further concern in using approaches involving modern genetics technology is the attitude of clinicians, patients and patient organizations as well as of the general public, regarding the use of genetic or clinical data and bio-banks for industrial studies. It is the same concerns that those raised by patients who are very willing to contribute to research that is academic and "non-commercial" but sometimes more reluctant to allow registers and bio-banks to be shared with industry. On the other hand, it is also quite difficult to proceed with the necessary research (notably expensive clinical trials) proving PM principles in any specific drug/disease study without the help of the pharmaceutical industry. Regulation for such collaborations is usually well looked into and it should become less of a concern and results in the promotion of PM orientated studies.

\section{Medical decisions}

For certain diseases the use of genotyping or genetic testing as a diagnostic tool or the prescription of drugs based on pharmacogenomic information is expected to become the standard of care in the near future. Physicians and pharmacists might be subject to liability if they lack sufficient knowledge of genetics to adequately interpret diagnostic tests and prescribe appropriate drug. The law in most countries has yet to establish the standard to which any physician will be held. Indeed, professionals have a duty to explain the risk of false-positive and false-negative test results and the implications of uncertainty of results [15], and in making disclosures, they are legally held to the high standard of knowledge in their specialty. In light of the current low level of instruction in genetics that is received in medical school, PM genetic counsellors might well become a novel specialty.

\section{Ethical}

From an ethical point of view, the usual criticism of genetic testing for disease diagnostic is that there is no proven direct medical benefit from the test. With complex diseases notably, conclusions can only be drawn from a genetic test for an individual, if the number of DNA markers tested positive for association with the disease is very high because each marker individually contributes very little to the disease. Psycho-social research has found little evidence that the results of a genetic test for a disease risk is prompting change behaviour towards sustained healthy or prevention from patients at high risk of a disease while there is as little evidence that it is bringing long-term negative, psychological impact [16].

In the context of PM (i.e., for response to therapy) such test is more likely to demonstrate direct benefit and therefore should raise less ethical concerns although it is also important to consider that PM offer a "chance" to select the right patient for the right drug at the right time but always with a certain percentage of risk to be wrong in the prediction of response as well as non-response. Therefore, rules may need to be established in order not to discriminate patients from receiving a specific drug due to a predictive test being negative and to lower any potential risk for inequities raised by the use of PM tools.

\section{Conclusion}

To achieve the promises of PM, a better understanding of its benefits and limitations needs to be developed at the level of the general public as well as at the level of individual patient. Conceptual, societal, ethical and cultural aspects of PM briefly reviewed in this paper, needs to be passed across at several levels involving all actors or the health system as well as patients and organisations. This will also reassure people that their genetic data is used properly in combination with advanced therapeutic protocols and drugs. Classification of patients according to the influences of genetic and environmental factors may lead to reliable conclusions about disease's susceptibility and clinical response. Thus, by inferring genetic characteristics obtained from a group of individuals to a single person, pharmacogenetics will be able to guide clinicians to prescribe the most appropriate drug. Therefore, patients must be aware of new treatments and issues concerning their efficacy, safety and potential adverse reactions, while doctors are expected to base treatment decision on the best available evidence but also considering the relative cost to benefit ratio of other available treatments.

\section{References}

1. Hudson KL, Rothenberg KH, Andrews LB, Kahn MJ, Collins FS (1995) Genetic discrimination and health insurance: an urgent need for reform. Science 270: 391-393.

2. McClellan KA, Avard D, Simard J, Knoppers BM (2013) Personalized medicine and access to health care: potential for inequitable access? Eur J Hum Genet 21: 143-147.

3. Calvo C, Johnson A (2001) (eds.) Genetics policy report: insurance issues. Washington DC: National Conference of State Legislatures.

4. Peterson EW, Finlayson M, Elliott SJ, Painter JA, Clemson L (2012) Unprecedented opportunities in fall prevention for occupational therapy practitioners. Am J Occup Ther 66: 127-130.

5. Lapham EV, Kozma C, Weiss JO (1996) Genetic discrimination: perspectives of consumers. Science 274: 621-624.

6. Clayton EW (2003) Ethical, legal, and social implications of genomic medicine. N Engl J Med 349: 562-569.

7. Durnin M, Hoy M, Ruse M (2012) Genetic testing and insurance: The complexity of adverse selection. Ethical Perspectives 19: 123-154.

8. Lander J, Van Hoyweghen I (2014) Streitkultur and the governance of genetic testing and insurance in Germany. New Genetics and Society 33: 42-59.

9. Pokorski RJ (1997) Insurance underwriting in the genetic era. Am J Hum Genet 60: 205-216.

10. Council of Europe: Additional Protocol to the Convention on Human Rights and Biomedicine, concerning Genetic Testing for Health Purposes 2008.

11. Ross LF, Saal HM, David KL, Anderson RR; American Academy of Pediatrics; American College of Medical Genetics and Genomics (2013) Technical report: Ethical and policy issues in genetic testing and screening of children. Genet Med 15: 234-245.

12. Sanderson SC, Wardle J (2008) Associations between anticipated reactions to genetic test results and interest in genetic testing: will selfselection reduce the potential for harm? Genet Test 12: 59-66.

13. Tercyak KP, Hensley Alford S, Emmons KM, Lipkus IM, Wilfond BS, et al. (2011) Parents' attitudes toward pediatric genetic testing for common disease risk. Pediatrics 127: e1288-1295.

14. Christenhusz GM, Devriendt K, Dierickx K (2013) To tell or not to tell? A systematic review of ethical reflections on incidental findings arising in genetics contexts. Eur J Hum Genet 21: 248-255.

15. Rothstein MA, Epps PG (2001) Ethical and legal implications of pharmacogenomics. Nat Rev Genet 2: 228-231.

16. McBride CM, Alford SH, Reid RJ, Larson EB, Baxevanis AD, et al. (2008) Putting science over supposition in the arena of personalized genomics. Nat Genet 40: 939-942. 